\title{
The Study on Features of Computational Thinking and Its Common Operation Mode Methods
}

\author{
Juan ZHONG ${ }^{1, \text { a }}$, Hua LIAO ${ }^{2, b}$ \\ ${ }^{1}$ College of Information Engineering, Gannan Medical University, Ganzhou, Jiangxi 341000,China \\ ${ }^{2}$ STATE GRID GANZHOU POWER SUPPLY COMPANY, Ganzhou, Jiangxi 341000,China \\ agyjuan1120@126.com
}

Keywords: Computational thinking; Recursive thinking; Separation of concerns; Worst case restore Heuristic reasoning.

\begin{abstract}
The computational thinking is the current international computer widely attention an important concept, is also the current computer education need to key research important topic. Computer industry at home and abroad in the field of philosophy and social science and the general scholars on this subject has carried on the extensive research and discussion. In this paper, the formation and development of computational thinking process has carried on the comprehensive analysis, to study the forming characteristics of computational thinking, focus on key content, summarizes the common patterns of computational thinking in combination with the existing research results and the research direction and its computational thinking is prospected in the face of challenges.
\end{abstract}

\section{Introduction}

Along with the advance of science, human being is not only the success of the content to know the world, and strength and speed of the world are also increasing. The world require application of existing knowledge, the design scheme can be implemented, reached the preset objectives, thus put forward the device, device, system and so on various aspects of the new design and manufacturing. And all of which emphasizes the constructive, realizability and verifiability, etc. These are all thinking of the new requirements and challenges for calculation [1]. Since industrial revolution of the 17th century, mankind from give priority to in order to know the world, turned to the rapid development of the world. The progress of the steam engine, electric power, material, medicine and so on thoroughly changed the world and people for the understanding of the world. In this process, the understanding of the nature into a constructive, the realizability of the new knowledge content, to create the nature of the object, it is human to deepen and continuation of knowledge application, in this process, computational thinking played an important role. Only the human understanding of natural law thinking into practical actions by calculation scheme, can achieve the goal of the world, can deepen the understanding of the original knowledge at the same time. The method of computational thinking is not only the world, but also to know the world. Along with the process of industrialization, people more and more about the importance of computational thinking clear understanding, also more and more benefit from computational thinking to bring fruitful results.

\section{Computational thinking summary}

Computational thinking is currently a high-profile involving computer problems and future direction of the basic concept of nature of science. This concept was first made by the Massachusetts institute of technology (MIT) Professor Seymour Papert in 1996 [2], but this a concept referred to the front, and is now attracted much attention is the representative of the United States Carnegie Mellon university (CMU) Jeannette M. Wing [3]. Computation thinking for problem solving a series of viewpoints and methods, these ideas and methods to help people more profound understanding of computing the essence and the core of the computer to solve the problem. Particularly useful for computer scientists and experts in the field of knowledge gap caused by the 
confusion. Natural and social problems of its own internal is richly contain belongs to calculate the evolution law of the evolution with the physical transformation, energy transformation and transformation of information. Therefore correctly extract the information transformation, and through the appropriate way to express it, make it be able to take advantage of computer processing of form, which is based on the concept of computational thinking of solving the problem of natural and social problems of the basic theory and methodology. Computer can't solve the problem of material transformation or energy conversion, but can use the abstract symbol transform to calculate, simulate and predict the evolution of the natural system and social system.

\section{The characteristics of computational thinking}

Thinking is calculated by using the basic concepts of computer science to solve problems, design system, and understanding of human behavior. It includes a series of thinking activity covers the breadth of computer science. When we have to solve a particular problem, how difficult it will first ask to solve this problem, what the best way to solve is. Computer science for accurately according to solid theory foundation to answer these questions. Describe the difficulty of the problem is the basic tool ability, must consider factors including machine instruction system, resource constraints, and operating environment. In order to effectively solve a problem, we may have to further ask whether an approximate solution is enough, whether can make use of a randomized, and whether to allow false positives and false negative. Computational thinking is through reduction, embedded, transformation and simulation methods, such as a question seems to be difficult to interpret as we know how to solve the problem [4-5]. In general, the characteristics of the computational thinking is shown in figure 1.

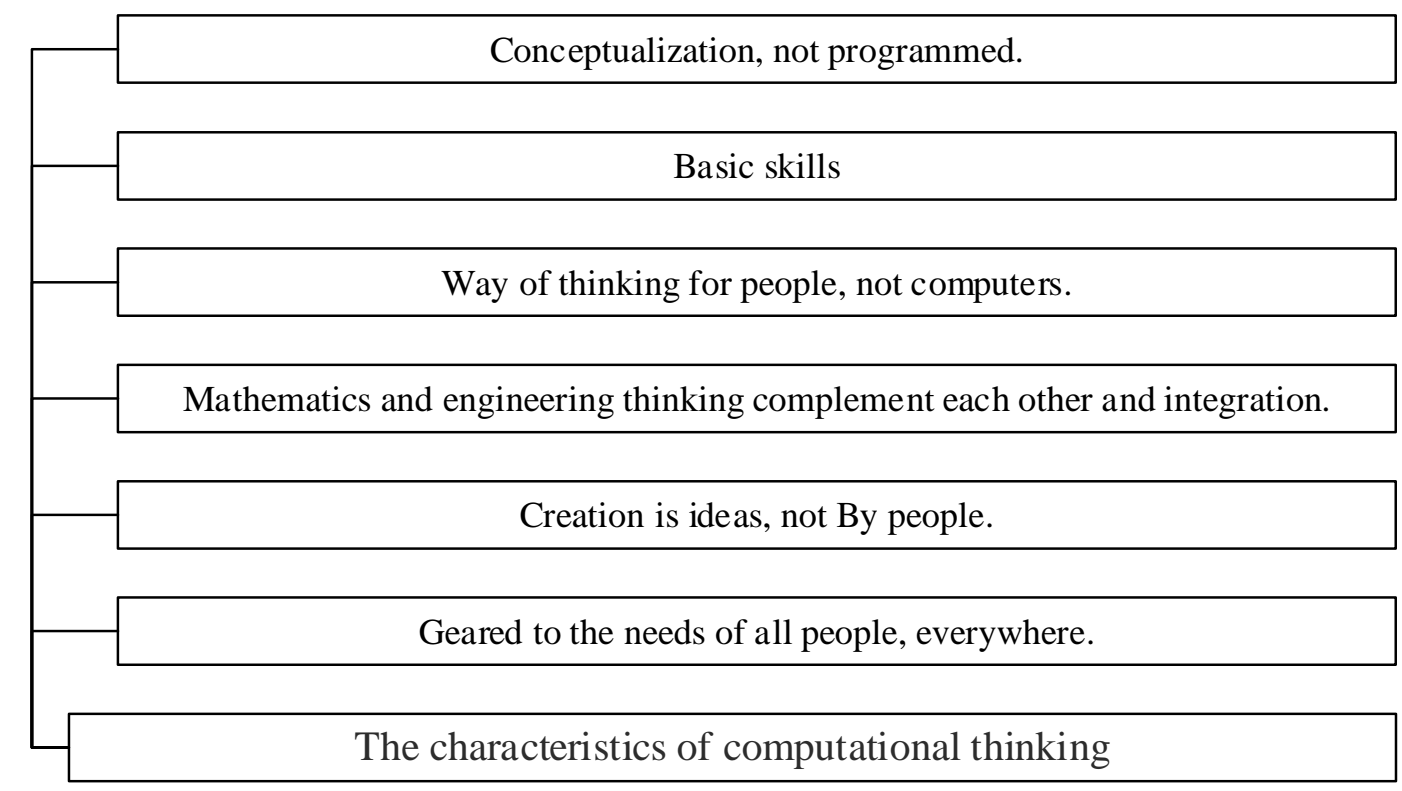

Figure 1. The characteristics of computational thinking

Conceptualization, not programmed. Computer science is not computer programming. Like a computer scientist to thinking means much more than for computer programming, can also require multiple layers on abstract thinking.

Basic skills. Basic skills is every individual to which in the modern society functions must master. Rigid skills means mechanical repetition. Ironically, when the computer think like a human thinking can really become mechanical.

Way of thinking for people, not computers. Computational thinking is a way of human to solve the problem, but it is by no means to make the human to think like computers. Computer dull and dreary, human intelligent and imaginative. It is the human to computer passion. Configuration 
computing devices, we can use our wisdom to solve those problems before the time of calculation did not dare to try, realize "less than" is only limited by region.

Mathematics and engineering thinking complement each other and integration. Computer science is essentially derived from mathematical thinking, because, like all of science, its formal foundation construction on mathematics. Computer science is essentially derived from engineering thinking, because we built is to be able to interact with the real world system, basic computing devices limit forced computer scientist must computational thinking, not just math thinking. Build a virtual world of freedom enables us to design various system beyond the physical world.

Creation is ideas, not by people. Not just our production software, hardware and others will be in the form of physical creation present everywhere and touch our lives, every minute and every second more importantly will have us to approach and solve problems, manage the calculation of daily life, communication and interaction with others

Geared to the needs of all people, everywhere. When calculating real thinking is integrated into the human activities that are no longer shown as a kind of explicit philosophy, it will become a reality.

\section{Commonly used computing thinking mode}

Computational thinking is the basic concept of human use of computer science to solve the problem, design system thinking activity, and understand the behavior the cloth covers computer science category. Commonly used computing thinking mode mainly includes four categories, as shown in figure 2 .

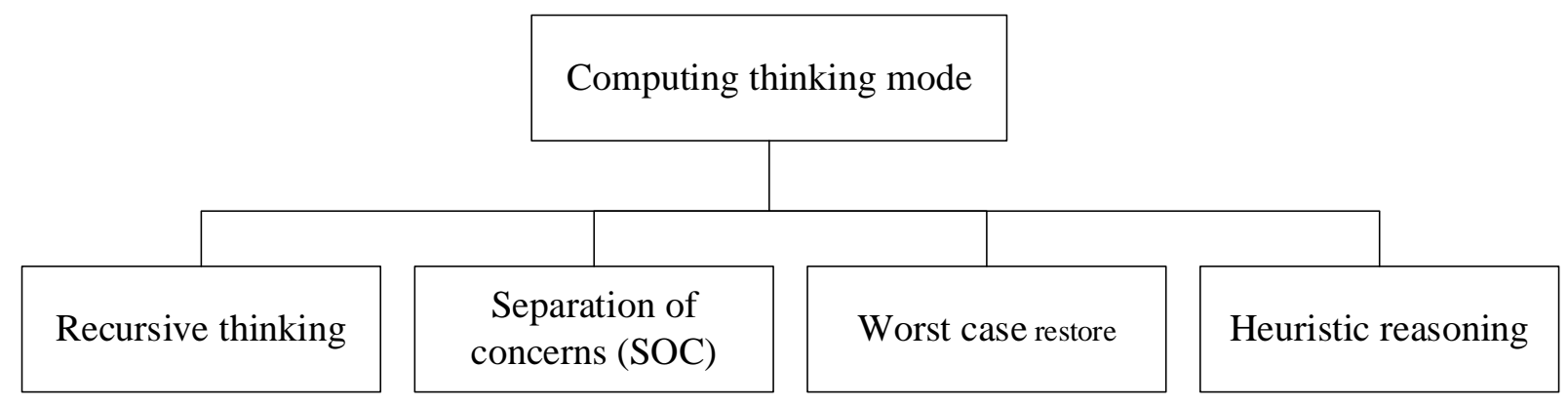

Figure 2. Commonly used computing thinking mode

Recursive thinking. It is a parallel processing. It is to put the code into the data and translate the data into the code. It was conducted by the generalized dimension analysis type checking. The alias or give multiple names of people and things, it is both know its benefits and understand its harm. For the method of indirect addressing and procedure call, it is know her power and understand its price. When evaluating a program, it not only according to the accuracy and efficiency, and aesthetic considerations, and for the design of the system, also considered concise and elegant.

Separation of concerns (SOC). It is associated to a particular concept, target software component are identified, encapsulation and manipulation ability, namely the ability of identification, encapsulation and manipulate your focus. Is a principle for dealing with complexity? Due to concerns mixed together can lead to complexity has greatly increased, so can bring different separation of concerns, the complexity of each treatment is a principle, a kind of method. Separation of concerns is the core concept of object-oriented programming. Separation of concerns that the code to solve the problem of specific area separate from the business logic and business logic code no longer contains code for domain specific problem calls (code for domain specific problem abstraction into less code, for example, the code in the function or a class), business logic and the problems in specific areas through side to encapsulate and maintenance, so that scattered in the changes in the entire application can very good management.

The worst case restore. According to the prevention, protection and through redundancy, fault tolerance and error correction way to recover from the worst case a thinking. It said jam for "dead 
lock", called the agreement "interface". Computational thinking is learning to meet each other in synchronous how to avoid "competitive conditions" (also called a "race conditions").

Heuristic reasoning. Is under the condition of uncertainty planning, learning, and scheduling. It is to search, search, search again, the result is a series of web pages, a strategy to win the game, or a counter example. Huge amounts of data is used to accelerate the calculation thinking, between time and space, make a trade-off between the processing power and storage capacity.

\section{Conclusion}

With the rise and development of computational thinking concept in our country, the computational thinking thoughts has been widely accepted by changing some theory and knowledge, the cultivation of thinking ability become one of the focus of educational research today. For computational thinking, to become a discipline, there is still a long way to go. At present, the calculation is not the subject of knowledge form of thinking, because of its concept, principle, characteristics, development methodologies, and knowledge innovation methodology system has not formed, nor most of the schools or the basic unit of the institute of teaching content. Scholars in this field, knowledge, information and academic information although materialization tissue is forming, but far did not reach maturity. In addition, the countries of the competent administrative department for education is still not fully realize the importance of computational thinking. Computational thinking subject system, therefore, to establish a long way to go.

\section{References}

[1] Benavides D, Segura S, Ruiz-Cortés A. Automated analysis of feature models 20 years later: A literature review[J]. Information Systems, 2010, 35(6): 615-636.

[2] Krippendorff K. Content analysis: An introduction to its methodology[M]. Sage, 2012.

[3] Denning P J. The profession of IT Beyond computational thinking[J]. Communications of the ACM, 2009, 52(6): 28-30.

[4] Zhang M, Ge Z, Song Z, et al. Global-local structure analysis model and its application for fault detection and identification[J]. Industrial \& Engineering Chemistry Research, 2011, 50(11): 6837-6848.

[5] Navlakha S, Bar - Joseph Z. Algorithms in nature: the convergence of systems biology and computational thinking[J]. Molecular systems biology, 2011, 7(1). 